\title{
Infusão contínua intravenosa de midazolam isolado ou associado ao fentanil para realização de endoscopia em suínos
}

\author{
Continuous infusion rate of midazolam alone or in combination with fentanyl for endoscopy in swine
}

\author{
Renata Navarro Cassu* Giulianne Carla Crociolli ${ }^{\mathrm{I}}$ Miriely Steim Diniz \\ Rafael Costa Guilhen ${ }^{I}$ Letícia Yamasaki ${ }^{I I}$
}

\section{RESUMO}

\begin{abstract}
Com este estudo objetivou-se avaliar o efeito cardiorrespiratório e a qualidade da anestesia $e$ da recuperação pós-anestésica decorrentes da associação cetamina e xilazina seguida da infusão contínua intravenosa (IV) de midazolam isolado ou associado ao fentanil, em suínos. Foram avaliadas 10 porcas adultas, da raça Landrace, com peso médio de $170 \pm 4 \mathrm{~kg}$, submetidas à endoscopia. Todos os animais foram medicados pela via intramuscular com cetamina $\left(4 \mathrm{mg} \mathrm{kg}^{-1}\right)$ associada à xilazina $\left(2 \mathrm{mg} \mathrm{kg}^{-1}\right)$. Vinte minutos após, foi realizado um bolus IV de cetamina (2mg $\left.\mathrm{kg}^{-1}\right)$, seguida da infusão contínua IV de midazolam $\left(0,5 \mathrm{mg} \mathrm{kg}^{-1} \mathrm{~h}^{-1} \mathrm{GM}, \mathrm{n=5}\right)$, ou midazolam $\left(0,25 \mathrm{mg} \mathrm{kg}^{-1} \mathrm{~h}^{-1}\right)$ associado ao fentanil $\left(4 \mu \mathrm{g} \mathrm{kg}^{-1}\right.$ $\left.h^{-1} G M F, n=5\right)$. Foram avaliados: frequência cardíaca $(F C) e$ ritmo cardíaco, pressão arterial sistólica (PAS), temperatura retal $(T)$, frequência respiratória $(f)$, variáveis hemogasométricas $\left(\mathrm{PaO}_{2}, \mathrm{PaCO}_{2}, \mathrm{pH}, \mathrm{HCO}_{3}^{-}, \mathrm{SaO}_{2}\right)$, qualidade da anestesia e qualidade e tempo da recuperação anestésica (RA). A análise estatística foi realizada por meio de análise de variância, teste de Tukey e o teste não paramétrico de Mann-Whitney $(P<0,05)$. A qualidade da anestesia foi semelhante entre os tratamentos, sendo possível a realização da endoscopia em ambos os grupos. Os parâmetros cardiorrespiratórios, bem como os gases sanguíneos, o pH, o bicarbonato e a saturação da oxihemoglobina não variaram entre os diferentes tratamentos. O tempo de recuperação anestésica foi de $98 \pm 15$ e $79 \pm 17$ minutos no GM e GMF, respectivamente, sem diferença entre os grupos. A qualidade da RA não diferiu significativamente entre os grupos. Conclui-se que ambos os tratamentos determinaram estabilidade cardiorrespiratória e foram satisfatórios para a realização de endoscopia em suínos. No entanto, apesar da ausência de diferença estatística, menor tempo de RA foi observado nos animais tratados com a infusão contínua de midazolam associado ao fentanil.
\end{abstract}

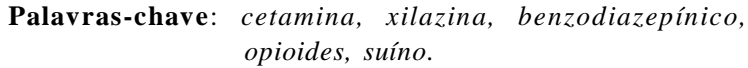

\begin{abstract}
The aim of this study was to evaluate the cardiopulmonary effects, the quality of anesthesia and the postanesthetic recovery of ketamine and xylazine premedication following intravenous (IV) continuous rate infusion of midazolam alone or in combination with fentanyl, in swine. Ten Landrace adult pigs that underwent endoscopy were evaluated. All pigs were premedicated with ketamine $\left(4 \mathrm{mg} \mathrm{kg}^{-1}\right)$ and xylazine $\left(2 \mathrm{mg} \mathrm{kg}^{-1}\right)$ by intramuscular administration. After twenty minutes, intravenous ketamine $\left(2 \mathrm{mg} \mathrm{kg}^{-1}\right)$ was injected, following IV continuous rate infusion of midazolam $\left(0.5 \mathrm{mg} \mathrm{kg}^{-1} \mathrm{~h}^{-1}\right.$ $G M, n=5)$ or midazolam $\left(0.25 \mathrm{mg} \mathrm{kg}^{-1} \mathrm{~h}^{-1}\right)$ plus fentanyl $(4 \mu \mathrm{g}$ $\left.\mathrm{kg}^{-1} \mathrm{~h}^{1} \mathrm{GMF}, \mathrm{n=5}\right)$. Heart and respiratory rates, systolic arterial blood pressure, rectal temperature, arterial blood gases, quality of anesthesia and recovery, and time for recovery were investigated. Statistical analysis was performed with One-Way ANOVA, Tukey test and Mann-Whitney test $(P<0.05)$. The quality of anesthesia was similar between treatments, and endoscopy was able to be performed in both groups. Cardiopulmonary variables and blood gases did not differ between groups. The recovery time was $98 \pm 15$ and $79 \pm 17$ minutes in GM and GMF, respectively, without significant difference between treatments. The quality of recovery did not differ between groups. The results allowed us to conclude that both treatments provide cardiopulmonary stability and were satisfactory to swine endoscopy. However, despite of absence of significant difference, shorter time of recovery was observed with midazolam/fentanyl infusion.
\end{abstract}

Key words: ketamine, xylazine, benzodiazepinics, opioids, pig.

'Departamento de Cirurgia e Anestesiologia Veterinária, Faculdade de Ciências Agrárias, Universidade do Oeste Paulista (Unoeste), Rodovia Raposo Tavares, Km 572, Campus II, Bairro Limoeiro, 19067-175, Presidente Prudente, SP, Brasil. E-mail: renavarro@uol.com.br.*Autor para correspondência.

IIDepartamento de Anatomia Patológica, Faculdade de Ciências Agrárias, Unoeste, Presidente Prudente SP, Brasil. 


\section{INTRODUÇÃO}

A endoscopia é uma técnica empregada no diagnóstico de enfermidades gastrointestinais e, apesar de não ser cruenta, pode causar desconforto, além de requerer imobilidade e relaxamento muscular (BARRIGA et al., 2008). No homem, para a realização da endoscopia, normalmente, são empregados fármacos miorrelaxantes, sedativos e analgésicos que não determinam a perda de consciência, exercendo pouca interferência no sistema cardiorrespiratório (LAZARAKI et al., 2007; HAYEE et al., 2009). Em animais, no entanto, um plano anestésico superficial é requerido, visto que a introdução do transdutor pela via oral pode determinar movimentos involuntários, bem como causar danos aos equipamentos e à equipe profissional (PÁPA et al., 2009).

Apesar da crescente utilização da espécie suína como modelo experimental, devido à semelhança com a espécie humana em diversos aspectos anatômicos e fisiológicos (SWINDLE \& SMITH, 1998), há escassez na literatura em relação aos protocolos anestésicos e sedativos indicados para realização de endoscopia em suínos.

Devido ao temperamento agressivo e à fácil indução de estresse, normalmente, os anestésicos dissociativos são empregados para a contenção química de suínos, visto a possibilidade da administração intramuscular desses fármacos (TENDILLO et al., 1996). Nessa espécie, a associação da xilazina à cetamina promove plano anestésico adequado, com analgesia e relaxamento muscular, sendo indicada para realização de procedimentos invasivos de curta duração. Em casos de procedimentos mais prolongados, doses adicionais de cetamina podem ser administradas pela via intravenosa, porém, as reaplicações prolongam a recuperação anestésica, sobretudo em animais cujo tecido adiposo é exuberante, como é o caso dos suínos adultos (LIN, 2007). Ademais, além de longa, a recuperação decorrente do uso de doses elevadas de cetamina pode ser agitada, predispondo o animal a traumatismos e estresse (AJADI et al., 2008).

Dessa forma, visando prolongar a sedação do animal em casos de procedimentos invasivos e não cruentos, como a endoscopia, ao invés da complementação anestésica com cetamina, pode-se optar pela administração coadjuvante de fármacos com propriedades ansiolíticas e/ou analgésicas que possibilitem maior relaxamento muscular, além de melhores condições para realização do exame diagnóstico, sem a necessidade de planos anestésicos mais profundos. Estudos prévios revelaram resultados satisfatórios com a infusão contínua de midazolam e fentanil em suínos sob anestesia geral, sendo observada estabilidade cardiovascular e redução da dose dos anestésicos utilizados (SØFTELAND et al., 1995; KAISER et al., 2003).

Este estudo teve como objetivo avaliar o efeito cardiorrespiratório, a qualidade da anestesia e o tempo e a qualidade da recuperação anestésica decorrentes da associação de cetamina e xilazina, seguida da infusão intravenosa contínua de midazolam isolado ou associado ao fentanil.

\section{MATERIAL E MÉTODO}

Foram avaliadas 10 porcas adultas da raça Landrace, com peso médio de $170 \pm 4 \mathrm{~kg}$, provenientes do Centro Zootécnico da Instituição de Origem, encaminhadas para realização de endoscopia para biópsia da mucosa gástrica. Após jejum sólido e hídrico de 24 e 12 horas, respectivamente, foi administrada, por via intramuscular, cetamina $\left(4 \mathrm{mg} \mathrm{kg}^{-1}\right)$ associada à xilazina $^{\mathrm{b}}\left(2 \mathrm{mg} \mathrm{kg}^{-1}\right)$. Vinte minutos após, a veia auricular foi cateterizada, sendo administrado um bolus adicional de cetamina $\left(2 \mathrm{mg} \mathrm{kg}^{-1}\right)$, seguido imediatamente da infusão contínua intravenosa de midazolam ${ }^{c}\left(0,5 \mathrm{mg} \mathrm{kg}^{1}\right.$ $\left.\mathrm{h}^{-1}, \mathrm{GM}, \mathrm{n}=5\right)$ ou midazolam $\left(0,25 \mathrm{mg} \mathrm{kg}^{-1} \mathrm{~h}^{-1}\right)$ associado ao fentanild $\left(4 \mu \mathrm{g} \mathrm{kg}^{-1} \mathrm{~h}^{-1}, \mathrm{GMF}, \mathrm{n}=5\right)$. Os fármacos foram diluídos em solução de Ringer lactato e administrados na taxa de $5 \mathrm{~mL} \mathrm{~kg}^{-1} \mathrm{~h}^{-1}$, mediante bomba de infusão peristáltica ${ }^{e}$. O volume referente aos fármacos administrados em infusão contínua foi adicionado ao Ringer lactato, após remoção de volume equivalente de soro, a fim de não alterar o volume final calculado na bomba de infusão.

A infusão contínua intravenosa foi mantida durante o período necessário para a realização da endoscopia. Terminado o procedimento, a infusão foi interrompida e o animal foi levado para a sala de recuperação anestésica, onde foi avaliado até o restabelecimento da posição quadrupedal.

Foram avaliados: frequência cardíaca (FC), por meio de eletrocardiograma ${ }^{\mathrm{f}}$, com eletrodos acoplados nos membros torácicos e pélvicos com leitura na segunda derivação de Eithoven; frequência respiratória $(f)$, por meio da inspeção dos movimentos torácicos; pressão arterial sistólica (PAS), com doppler vascularg, mediante a adaptação do manguito na cauda do animal, respeitando-se uma relação de 0,4 entre a largura do manguito e a circunferência da cauda; gases sanguíneos $\left(\mathrm{PaCO}_{2} \mathrm{PaO}_{2}\right), \mathrm{pH}, \mathrm{SaO}_{2}$ e bicarbonato, por punção da artéria femoral, em seringas previamente heparinizadas, com leitura imediata no hemogasômetro ${ }^{\mathrm{h}}$, que foi ajustado em função da temperatura retal (T) do 
animal, registrada no momento da colheita da amostra sanguínea, mediante a introdução do termômetro digital ${ }^{i}$ na ampola retal, durante dois minutos.

Esses parâmetros foram avaliados aos 5, 10, 20 e 30 minutos após o início das infusões IV, com exceção da hemogasometria, a qual foi realizada apenas aos 5 e 30 minutos.

A qualidade da anestesia foi avaliada em função da reação do animal perante o procedimento, com método de escore, conforme descrito em estudo prévio (WALKER et al., 2004), em que: $0=$ nenhum movimento ou reação frente ao procedimento; $1=$ discreta movimentação de membros e/ou cabeça; $2=$ movimentação de membros e/ou cabeça e vocalização; 3 =intensa vocalização e movimentos bruscos de membros e/ou cabeça. Os animais cujos escores fossem maior ou igual a dois receberiam incremento do plano anestésico por meio da administração intravenosa em bolus de $2 \mathrm{mg} \mathrm{kg}^{-1}$ de cetamina.

A qualidade da recuperação anestésica (RA) foi avaliada em função do comportamento do animal (Tabela 1), conforme proposto em estudo prévio (AJADI et al., 2008). Também foram avaliados, em minutos, os tempos do procedimento e da RA, sendo esta compreendida a partir da interrupção da infusão contínua dos fármacos até o restabelecimento da posição quadrupedal.

A estatística foi feita com Análise de Variância de uma via (One-way ANOVA), seguida pelo teste de Tukey. Para as medianas de escore entre grupos, foi utilizado o teste não paramétrico de Mann-Whitney. As análises foram realizadas empregando-se programa Graphpad Instat, com nível de significância de 5\%.

\section{RESULTADOS}

Com relação às variáveis cardiovasculares, não houve diferença entre os grupos em relação à $\mathrm{FC} \mathrm{e}$ à PAS ao longo do tempo. Na comparação entre momentos, a FC foi superior aos 5 e 10 minutos em relação aos 20 e 30 minutos após o início da infusão de midazolam ou midazolam/fentanil (Tabela 2).

A $f$ não diferiu entre os grupos ao longo do tempo e nem entre os momentos dentro de um mesmo grupo. Comportamento semelhante foi observado em relação ao pH, $\mathrm{PaCO}_{2} \mathrm{PaO}_{2}, \mathrm{pH}, \mathrm{SaO}_{2}$ e $\mathrm{HCO}_{3}$ (Tabela 2).

A T não variou entre os grupos ao longo do tempo. Na comparação entre momentos, valores superiores foram observados aos 5 e 10 minutos em relação aos 30 minutos após o início da infusão, para ambos os tratamentos (Tabela 2).

A qualidade da anestesia não variou significativamente entre os tratamentos, sendo atribuído escore 0 para todos os animais, de modo que nenhum deles demonstrou qualquer tipo de reação frente à passagem do transdutor, não havendo a necessidade de suplementação anestésica, em ambos os grupos. O tempo para realização da endoscopia não variou entre os grupos, com duração média de $31 \pm 5$ e $29 \pm 6$ minutos no GM e GMF, respectivamente.

O tempo de RA não diferiu de forma significativa entre os grupos, sendo de $98 \pm 15$ e $79 \pm 17$ minutos no GM e GMF, respectivamente. Com relação à qualidade da RA, não houve diferença significativa entre os tratamentos, porém, foram atribuídos escores 0 e 2 em $100 \%$ dos animais do GM e GMF, respectivamente, ao adquirirem a posição esternal.

Tabela 1 - Critérios empregados na avaliação da qualidade da recuperação anestésica de suínos após infusão contínua intravenosa de midazolam isolado (GM) ou associado ao fentanil (GMF) e número de animais classificados de cada categoria em cada grupo.

\begin{tabular}{|c|c|c|c|}
\hline \multirow[t]{2}{*}{ Escore } & \multirow[t]{2}{*}{ Comportamento em decúbito lateral } & \multicolumn{2}{|c|}{ Número de animais } \\
\hline & & GM $(n=5)$ & GMF $(n=5)$ \\
\hline 0 & Animal quieto, ocasionalmente movimenta pernas ou levanta a cabeça. & 5 & 5 \\
\hline 1 & Animal ansioso, com movimentos de pedalagem. & & \\
\hline 2 & $\begin{array}{l}\text { Animal com sinais de euforia: vocalização, pedalagem intensa. } \\
\text { Comportamento para adquirir a posição esternal. }\end{array}$ & & \\
\hline 0 & Movimento suave e com coordenação & 5 & \\
\hline 1 & Leve agitação. & & \\
\hline 2 & $\begin{array}{l}\text { Mais que uma tentativa para o decúbito esternal. } \\
\text { Comportamento para adquirir a posição quadrupedal. }\end{array}$ & & 5 \\
\hline 0 & Posição apropriada para os quatro membros, capacidade de andar na primeira tentativa. & 1 & 1 \\
\hline 1 & $\begin{array}{l}\text { Capacidade de levantar-se, porém, fraqueza nos membros, com grau discreto a } \\
\text { moderado de ataxia. }\end{array}$ & & 1 \\
\hline 2 & Mais que uma tentativa para ficar em pé, com intensa ataxia. & 4 & 3 \\
\hline
\end{tabular}

Adaptação de escore de recuperação pós-anestésica proposto por AJADI et al. (2008).

Ciência Rural, v.42, n.12, dez, 2012. 
Tabela 2 - Valores médios e desvios-padrão da frequência cardíaca (FC), frequência respiratória $(f)$, pressão parcial arterial de dióxido de carbono $\left(\mathrm{PaCO}_{2}\right)$, pressão parcial arterial de oxigênio $\left(\mathrm{PaO}_{2}\right), \mathrm{pH}$ arterial, saturação arterial de oxigênio na hemoglobina $\left(\mathrm{SaO}_{2}\right)$, bicarbonato arterial $\left(\mathrm{HCO}_{3}{ }^{-}\right)$, pressão arterial sistólica (PAS) e temperatura retal (T) em suínos sob infusão contínua intravenosa de midazolam isolado (GM, n=5) ou associado ao fentanil (GMF, $n=5)$.

\begin{tabular}{|c|c|c|c|c|}
\hline Grupos & $5 \mathrm{~min}$ & $10 \mathrm{~min}$ & $20 \mathrm{~min}$ & $30 \mathrm{~min}$ \\
\hline \multicolumn{5}{|c|}{-- } \\
\hline GM & $98 \pm 24$ & $95 \pm 32$ & $84 \pm 26^{*}$ & $80 \pm 22 *$ \\
\hline GMF & $80 \pm 23$ & $77 \pm 23$ & $67 \pm 26^{*}$ & $64 \pm 26^{*}$ \\
\hline & & $f(\operatorname{mov} \mathrm{mi}$ & 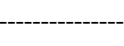 & 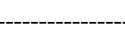 \\
\hline GM & $24 \pm 3$ & $25 \pm 3$ & $24 \pm 8$ & $26 \pm 6$ \\
\hline GMF & $23 \pm 7$ & $28 \pm 7$ & $26 \pm 8$ & $27 \pm 9$ \\
\hline ------.. & & $\mathrm{aCO}_{2}(\mathrm{mn}$ & & \\
\hline GM & $33 \pm 4$ & ---------- & ----------- & $36 \pm 2$ \\
\hline GMF & $32 \pm 5$ & --------- & ---------- & $33 \pm 3$ \\
\hline GM & $91+9$ & $\mathrm{PaO}_{2}(\mathrm{~mm}$ & - & $100+10$ \\
\hline GMF & $94 \pm 3$ & -------- & ---------- & $100 \pm 11$ \\
\hline GM & $7,37 \pm 0,07$ & ----- & ---------- & $7,42 \pm 0,02$ \\
\hline GMF & $7,38 \pm 0,10$ & -------- & --------- & $7,44 \pm 0,03$ \\
\hline GM & $98 \pm 0,02$ & -------- & --------- & $98 \pm 0,03$ \\
\hline GMF & $97 \pm 0,02$ & -------- & ---------- & $97 \pm 0,03$ \\
\hline & 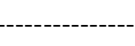 & $\mathrm{HCO}_{3}^{-}(\mathrm{mE}$ & & \\
\hline GM & $21,8 \pm 3$ & -------- & -------- & $21,2 \pm 4$ \\
\hline GMF & $22 \pm 2$ & ---------- & ---------- & $21,7 \pm 3$ \\
\hline GM & $114 \pm 16$ & $\begin{array}{c}\text { PAS (mm } \\
113 \pm 15\end{array}$ & $109 \pm 14$ & $118 \pm 15$ \\
\hline GMF & $110 \pm 13$ & $109 \pm 14$ & $112 \pm 10$ & $113 \pm 4$ \\
\hline GM & $38,6 \pm 0,3$ & $\begin{array}{l}--\mathrm{T}(\mathrm{C}) \\
38,4 \pm 0,2\end{array}$ & $38,0 \pm 0,2$ & $37,6 \pm 0,2 *$ \\
\hline GMF & $38,5 \pm 1,1$ & $38,3 \pm 0,8$ & $38,1 \pm 0,4$ & $37,4 \pm 0,3 *$ \\
\hline
\end{tabular}

*P $<0,05$ redução significativa em relação aos 5 e 10 minutos após o início da infusão intravenosa de midazolam (GM) e midazolam/fentanil (GMF).

Paralelamente, foram registrados escores de 0 e 2 em $20 \%$ e $80 \%$, respectivamente, nos animais do GM e escores 0,1 e 2 em $20 \%, 20 \%$ e $60 \%$, respectivamente, nos animais do GMF ao adquirirem a posição quadrupedal (Tabela 1).

\section{DISCUSSÃO}

Os achados do atual estudo evidenciaram a viabilidade da infusão contínua IV de midazolam isolado ou associado ao fentanil para a realização da endoscopia para colheita de amostra por meio de biópsia gástrica, corroborando resultados prévios relatados no homem (LAZARAKI et al., 2007; BARRIGA et al., 2008; HAYEE et al., 2009).

Neste estudo, a dose do midazolam foi reduzida em $50 \%$, quando associado ao fentanil, no entanto, o efeito sedativo não foi prejudicado, bem como o grau de relaxamento muscular, visto que a endoscopia foi realizada com sucesso em todos os animais sem a necessidade de suplementação anestésica.

Todavia, os animais tratados com midazolam/fentanil apresentaram menor tempo de RA (79 \pm 17 minutos) em comparação aos tratados com a infusão de midazolam isolado ( $98 \pm 15$ minutos), confirmando resultados observados no homem que demonstraram recuperação mais tardia com a infusão contínua de midazolam em relação à infusão de fentanil (LAZARAKI et al., 2007; CORREIA et al., 2011). Dessa forma, sugere-se que a redução da dose do midazolam em $50 \%$ e a rápida meia vida de eliminação do fentanil favoreceu a RA dos animais tratados com a combinação desses fármacos. Estudos desenvolvidos em suínos 
revelaram que a meia vida de eliminação do fentanil $\left(50 \mu \mathrm{g} \mathrm{kg}^{-1}\right)$ foi de $35,8 \pm 8,2$ minutos após a administração IV (SHIEBER et al., 1985), enquanto que a meia vida de eliminação do midazolam $\left(0,4 \mathrm{mg} \mathrm{kg}^{-1} \mathrm{IV}\right)$ foi de $158 \pm 127$ minutos (LACOSTE et al., 2000).

Paralelamente, embora a qualidade da RA não tenha diferido de forma significativa entre os tratamentos, o comportamento dos animais foi variável nas diferentes fases da recuperação. Na fase inicial da RA, enquanto em decúbito lateral, perfil semelhante foi observado em ambos os tratamentos, com comportamento calmo em todos os animais, refletindo grau de sedação intenso, provavelmente, devido à elevada concentração plasmática dos fármacos administrados sob infusão contínua. Contudo, na fase intermediária da RA, observou-se que o grupo tratado com midazolam/fentanil apresentou dificuldade para assumir a posição esternal, sendo atribuído escore 2 em $100 \%$ dos animais, enquanto que os animais tratados com midazolam isolado foram capazes de assumir prontamente a posição esternal. É possível que, nessa fase, a concentração plasmática do fentanil ainda fosse suficiente para potencializar o efeito ansiolítico do midazolam, resultando em grau de sedação mais intenso, quando comparado ao grupo tratado com midazolam isolado. Já na fase final da RA, maior incidência de ataxia e tentativas para a obtenção da posição quadrupedal foram constatadas nos animais tratados com midazolam isolado, provavelmente, devido ao declínio da concentração plasmática do fentanil, resultante da rápida redistribuição tecidual, em função de sua elevada lipossolubilidade (RAJAN et al., 1998). Em cães mantidos sob infusão contínua de fentanil $\left(10 \mu \mathrm{g} \mathrm{kg}^{-1} \mathrm{~min}^{-1}\right)$ durante 60 minutos, foi constatada redução significativa da concentração plasmática 20 minutos após a interrupção da administração (SANO et al., 2006). Ademais, sugere-se que a redução da dose do midazolam em $50 \%$ no tratamento GMF também favoreceu a menor incidência de ataxia (60\% no GMF versus $80 \%$ do GM). Em pacientes humanos encaminhados para endoscopia gastrointestinal, foi relatada maior interferência nas respostas cognitivas e motoras com a associação do midazolam em relação aos opioides nos protocolos de sedação (VANNATTA\& REX, 2006).

Com relação à $\mathrm{FC}$, apesar da ausência de diferença estatística entre os grupos, valores médios mais elevados foram observados nos animais tratados com midazolam isolado, bem como bradicardia só foi detectada no grupo tratado com midazolam/fentanil, sendo constatada FC abaixo de 60bat $\mathrm{min}^{-1} \mathrm{em}$ dois animais. A FC é controlada essencialmente por um componente que se origina de neurônios cardíacos vagais parassimpáticos no núcleo ambíguo, situado na medula, que fazem sinapse com gânglios localizados na base do coração. $\mathrm{O}$ fentanil reduz a frequência e a amplitude da transmissão GABAérgica para esses neurônios cardíacos vagais, culminando com a redução da FC, conforme já demonstrado por outros autores (SHIEBER et al., 1985; GRIFFIOEN et al., 2004). Nos primeiros momentos de avaliação (5 e 10 minutos), a FC foi mais elevada em relação aos 20 e 30 minutos após o início da infusão contínua em ambos os tratamentos, provavelmente em função do efeito simpatomimético determinado pela cetamina, cujo período de ação é de aproximadamente 15 minutos após administração intravenosa (LIN, 2007). Ademais, o midazolam reduz a FC em aproximadamente $20 \% \mathrm{em}$ suínos (SMITH et al., 1991), enquanto o fentanil reduz em torno de 36\% (SHIEBER et al., 1985), de modo que, ao longo da infusão desses fármacos, o efeito cronotrópico negativo tornou-se mais pronunciado.

Estabilidade foi observada na PAS em ambos os grupos, com valores dentro dos limites fisiológicos para a espécie (PAS $100-150 \mathrm{mmHg}$ ) (HASKINS, 2007), corroborando estudos prévios sobre o uso da infusão contínua de midazolam associado ao fentanil em suínos (SØFTELAND et al., 1995; KAISER et al., 2003). Embora a cetamina possa determinar o aumento de até $25 \%$ na pressão arterial em função de sua atividade simpatomimética indireta (LIN, 2007), esse efeito foi atenuado em razão da baixa dose empregada desse anestésico (total de $6 \mathrm{mg} \mathrm{kg}^{-1}$ ), favorecendo a estabilidade dessa variável, visto que tanto o midazolam quanto o fentanil exercem pouca interferência sobre a pressão arterial (SHIEBER et al., 1985; SMITHet al., 1991).

A discreta redução da T observada ao longo do tempo pode ser atribuída ao efeito depressor central, induzido por ambos os tratamentos, de modo a interferir sobre os mecanismos termorreguladores do hipotálamo (HASKINS, 2007). No entanto, apesar dos valores inferiores observados ao término do procedimento em relação aos valores iniciais (5 e 10 minutos após início da infusão), este achado não teve relevância clínica, em virtude da manutenção da $\mathrm{T}$ dentro dos limites fisiológicos para a espécie.

Com relação ao sistema respiratório, a $f$, o $\mathrm{pH}$ e os gases sanguíneos mantiveram-se estáveis durante todo o procedimento em ambos os grupos, confirmando resultados prévios que evidenciaram pouca interferência sobre a dinâmica respiratória com a infusão contínua de midazolam e fentanil em suínos (SØFTELAND et al., 1995). SMITH et al. (1991) demonstraram redução de $50 \%$ da $f$ em relação aos valores basais após administração intramuscular de $0,1 \mathrm{mg} \mathrm{kg}^{-1}$ de midazolam em suínos, contudo, não foram 
detectadas alterações no $\mathrm{pH}$ e nos gases sanguíneos. Já o fentanil, por atuar nos receptores opioides $\mu$, pode induzir depressão dose-dependente da função respiratória, caracterizada pelo aumento da $\mathrm{PaCO}_{2} \mathrm{e}$ redução da $\mathrm{PaO}_{2}$ (SCHIEBER et al., 1985). Em suínos, a infusão contínua IV de fentanil $\left(10 \mu \mathrm{g} \mathrm{kg}^{-1} \mathrm{~h}^{-1}\right)$, mantida durante seis horas, resultou em redução na $\mathrm{PaO}_{2}$ somente a partir da segunda hora de infusão, sugerindo a necessidade de suporte de oxigênio em casos de tratamento por período prolongado (RAJAN et al., 1998).

Paralelamente, deve-se ressaltar que a xilazina também exerce efeitos sedativos, analgésicos e cardiorrespiratórios, podendo ser observada a ocorrência de bradiarritmias, hipertensão transitória e depressão dose-dependente da função respiratória (AJADI et al., 2008). Entretanto, no atual estudo, apenas uma dose de xilazina foi aplicada 20 minutos antes da injeção IV de cetamina, de modo que o intervalo entre a administração da xilazina e o início da mensuração paramétrica foi de $30 \pm 12$ minutos. Dessa forma, sugerese que a xilazina determinou mínima interferência nos resultados do presente estudo, em função do curto período de ação desse fármaco na espécie suína (TENDILLO et al., 1996).

\section{CONCLUSÃO}

Conclui-se que ambos os tratamentos foram satisfatórios para a realização de endoscopia em porcas adultas, além de determinarem estabilidade cardiorrespiratória, com exceção da ocorrência de bradicardia observada em dois animais do GMF. No entanto, apesar da ausência de diferença estatística, menor tempo de RA foi observado nos animais tratados com a infusão contínua de midazolam associado ao fentanil.

\section{FONTES DE AQUISIÇÃO}

a-Cetamin 10\%, Syntec do Brasil, Cotia, SP, Brasil. b-Xilazina 10\%, Vetec S.A., Movil, Buenos Aires, Argentina. c-Midazolam, União Química Farmacêutica Nacional S/A, São Paulo, SP, Brasil.

d-Fentanest, Cristália Produtos Químicos e Farmacêuticos Ltda, Itapira, SP, Brasil.

e-Bomba linear ST1000, Samtronic Infusion Systems, São Paulo, SP, Brasil.

f-ECG PC Eletrocardiógrafo Digital, Tecnologia Eletrônica Brasileira (TEB), São Paulo, SP, Brasil.

g-Doppler 841-A, Parks Medical Electronics, Las Vegas, NV, USA.

h-248-pH Blood Gas Analyser, Ciba Corning Diagnostics, Medfield, MA, USA.

i-Termômetro digital, Becton Dickinson do Brasil, São Paulo, SP, Brasil.

\section{COMITÊ DE ÉTICA}

Este estudo foi aprovado pela Comissão de Ética de Uso de Animais na Experimentação (CEUA) da Instituição de Origem (protocolo n.58/09) e realizado de acordo com os princípios éticos na experimentação animal.

\section{REFERÊNCIAS}

AJADI, R.A. et al. Increasing ketamine dose enhances the anaesthetic properties of ketamine-xylazine-midazolam combinations in growing pigs. Journal of the South African Veterinary Association, v.79, p.205-207, 2008. Disponível em: 〈http://www3.interscience.wiley.com>. Acesso em: 10 abr. 2009. doi: 10.1111/j.1467-2995.2009.00496.x.

BARRIGA, J. et al. Sedation for upper endoscopy: comparison of midazolam versus fentanyl plus midazolam. Southern Medical Journal, v.101, p.362-366, 2008.

CORREIA, L.M. et al. Sedation during upper GI endoscopy in cirrhotic outpatients: a randomized, controlled trial comparing propofol and fentanyl with midazolam and fentanyl. Gastrointestinal Endoscopy, v.73, p.45-51, 2011.

GRIFFIOEN, K.J. et al. Fentanyl inhibits GABAergic neurotransmission to cardiac vagal neurons in the nucleus ambiguus. Brain Research, v.1007, n.1-2, p.109-115, 2004.

HASKINS, S.C. Monitoring anesthetized patients. In: TRANQUILLI, W.J. et al. Lumb \& Jones' veterinary anesthesia and analgesia. 4.ed. Oxford: Blackwell Publishing, 2007. Cap.19, p.533-560.

HAYEE, B. et al. Midazolam with meperidine or fentanyl for colonoscopy: results of a randomized trial. Gastrointestinal Endoscopy, v.69, p. 681-687, 2009. Disponível em: <http:// www3.interscience.wiley.com>. Acesso em: 10 abr. 2009. doi: 10.1016/j.gie.2008.09.033.

KAISER, G.M. et al. Intravenous infusion anesthesia with propofol-midazolam-fentanyl for experimental surgery in swine. Journal of Investigative Surgery: the Official Journal of the Academy of Surgical Research, v.16, p.353-357, 2003. Disponível em: <http://www.ncbi.nlm.nih.gov/pubmed>. Acesso em: 18 set. 2010. doi: 10.1080/08941930390250223.

LACOSTE, L. et al. Intranasal midazolam in piglets: pharmacodynamics $\left(0.2\right.$ vs $\left.0.4 \mathrm{mg} \mathrm{kg}^{-1}\right)$ and pharmacokinetics $\left(0.4 \mathrm{mg} \mathrm{kg}^{-1}\right)$ with bioavailability determination. Laboratorial Animal, v.34, p.29-35, 2000.

LAZARAKI, G. et al. Single use of fentanyl in colonoscopy is safe and effective and significantly shortens recovery time. Surgery and Endoscopy, v.21, p.1631-1636, 2007. Disponível em: 〈http://www.ncbi.nlm.nih.gov/pubmed〉. Acesso em 18 set. 2010. doi: 10.1007/s00464-007-9215-y.

LIN, H. Dissociative anesthetics. In: TRANQUILLI, W.J. et al. Lumb \& Jones' veterinary anesthesia and analgesia. 4.ed. Oxford: Blackwell Publishing, 2007. Cap.12, p.301-354.

PÁPA, K. et al. Endoscopically guided nasojejunal tube placement in $\operatorname{dog}$ s for short-term postduodenal feeding. Journal of Veterinary Emergency and Critical Care, v.19, p.554-563, 
2009. Disponível em: 〈http://www3.interscience.wiley.com〉. Acesso em: 08 jun. 2010. doi: 10.1111/j. 14764431.2009.00477.x.

RAJAN, V. et al. Pharmacodynamic effects and pharmacokinetic profile of continuous infusion fentanyl in newborn piglets. Biology of the Neonate, v.74, p.39-47, 1998.

SANO, T. et al. Pharmacokinetics of fentanyl after single intravenous injection and constant rate infusion in dogs. Veterinary Anaesthesia and Analgesia, v.33, p.266-273, 2006. Disponível em: 〈http://www.ncbi.nlm.nih.gov/pubmed>. Acesso em: 27 mar. 2012. doi: 10.1111/j.14672995.2005.00266.x.

SCHIEBER, R.A. et al. Cardiovascular and pharmacodynamic effects of high-dose fentanyl in newborn piglets. Anesthesiology, v.2, p.166-171, 1985.

SMITH, A.C. et al. Sedative and cardiovascular effects of midazolam in swine. Laboratory Animal Science, v.2, 157$161,1991$.
SØFTELAND, E. et al. Evaluation of thiopentone-midazolamfentanyl anaesthesia in pigs. Laboratory Animals, v.29, p.269-275, 1995.

SWINDLE, M.M.; SMITH, A.C. Comparative anatomy and physiology of the pig. Scandinavian Journal of Laboratory Animal Science, v.25, p.11-21, 1998.

TENDILLO, F.J. et al. Cardiopulmonary and analgesic effects of xylazine, detomidine, medetomidine, and the antagonist atipamezole in isoflurane-anesthetized swine. Laboratory Animal Science, v.46, p.215-219, 1996.

VANNATTA, M.; REX, D. Propofol alone titrated to deep sedation versus propofol in combination with opioids and/or benzodiazepines and titrated to moderate sedation for colonoscopy. American Journal of Gastroenterology, v.101, p.2209-2217, 2006.

WALKER, B. et al. Inhalation anaesthesia for castration of newborn piglets: experiences with isoflurane and isoflurane/ NO. Journal of Veterinary Medicine. A Physiology, Pathology, Clinical Medicine, v.51, p.150-154, 2004. 